\title{
Atividade antifúngica de extratos hidroalcoólicos em isolados de Candida obtidos de secreção vaginal.
}

\author{
Hydroalcoholic extracts antifungal activity against Candida sp. obtained from \\ vaginal secretion.
}

\author{
Camilla Itapary dos Santos ${ }^{1}$; Cristina de Andrade Monteiro ${ }^{1,2}$
}

\begin{abstract}
Resumo: Introdução: Candidíase vulvovaginal (CVV) é o nome dado ao quadro infeccioso causado por leveduras do gênero Candida, caso não seja feito tratamento, CVV pode evoluir para outros problemas de saúde. A frequência de isolados clínicos resistentes aos agentes antifúngicos utilizados vem aumentando ao longo dos anos, por esse motivo, o presente trabalho buscou investigar a atividade antifúngica de algumas plantas contra isolados de Candida spp obtidos de cavidade vaginal. Material e métodos: Os extratos hidroalcóolicos obtidos de Psidium spp, Cecropia spp, Byrsonima spp, Rosmarinus spp e Cucurbita spp foram preparados à partir do método de maceração em álcool $50 \%$, durante 10 dias. Em seguida foram testados em isolados de secreção vaginal de $C$. albicans, $C$. tropicalis, $C$. parapsilosis e $C$. glabrata, através dos métodos de difusão em Ágar e microdiluição. Resultados: O extrato de Psidium spp apresentou atividade no teste de difusão, formando halos de $1,4 \mathrm{~cm}$ e $1,8 \mathrm{~cm}$ em linhagens de $C$. tropicalis. No teste em meio líquido todos os extratos foram capazes de inibir o crescimento de Candida spp. O teste de microdiluição mostrou que o extrato de Cecropia spp foi capaz de inibir o crescimento de $C$. tropicalis em $58 \%$. Todos os extratos testados apresentaram atividade contra Candida e, portanto, são necessários mais ensaios para avaliar a segurança de aplicação desses extratos. Conclusão: Todos os extratos apresentaram capacidade de inibir o crescimento de Candida no teste de microdiluição.
\end{abstract}

Palavras-chave: Psidium, Cecropia, Byrsonima, Rosmarinus, Cucurbita, anti-candida

\begin{abstract}
Introduction: Candidiasis vulvovaginal (CVV) is the name given to the infectious condition caused by yeasts of the genus Candida, and if not treated, CVV may progress to other health problems. The frequency of clinical isolates resistant to the antifungal agents used has been increasing over the years. Therefore, the present work aimed to investigate the antifungal activity of some plants against Candida spp isolates obtained from vaginal cavity. Material and methods: The hydroalcoholic extracts obtained from Psidium spp., Cecropia spp., Byrsonima spp., Rosmarinus spp. and Cucurbita spp were prepared from the $50 \%$ alcohol maceration method for 10 days. They were then tested in isolates of vaginal secretion of $C$. albicans, $C$. tropicalis, C. parapsilosis and C. glabrata, using diffusion methods in agar and microdilution. Results: The extract of Psidium spp presented activity in the diffusion test, forming inhibition zones of $1.4 \mathrm{~cm}$ and $1.8 \mathrm{~cm}$ in $C$. tropicalis strains. In the microdilution test all extracts were able to inhibit the growth of Candida spp. The microdilution test showed that the extract of Cecropia spp was able to inhibit the growth of $C$. tropicalis in $58 \%$. All extracts tested showed activity against Candida and, therefore, more tests are needed to evaluate the safety of application of these extracts. Conclusion: All hidroalchoolic extracts were able to inhibit Candida growing in microdilution test.
\end{abstract}

Key-words: Psidium, Cecropia, Byrsonima, Rosmarinus, Cucurbita, anti-candida

\footnotetext{
${ }^{1}$ Universidade CEUMA. Rua Josué Montello, n.1 - Renascença II, São Luís - MA, 65075-120. Telefone: 32144333. E-mail: camilla itapary@hotmail.com

2 Instituto Federal do Maranhão
} 


\section{Introdução}

Candidíase vulvovaginal (CVV) é o nome dado ao quadro infeccioso causado por leveduras do gênero Candida e é considerado o segundo tipo mais comum de infecção vaginal em mulheres com idade reprodutiva ${ }^{1}$. Apresenta entre os principais sintomas: prurido, edema, dispareunia, presença de corrimento com aspecto de "queijo coalhado" e alguns fatores como gravidez, antibioticoterapia, uso de contraceptivos, diabetes e imunossupressão podem ser predisponentes para o aparecimento da infecção ${ }^{2}$. Se não for tratada, CVV pode contribuir para o aparecimento de outros problemas de saúde na paciente como: risco de infertilidade, doença inflamatória pélvica, gravidez ectópica, aborto, desordens no fluxo menstrual, parto prematuro, candidíase cutânea congênita, aumento da susceptibilidade de infecção por HIV ${ }^{1,2,3,4}$. Normalmente a infecção é causada por $C$. albicans, entretanto, $O$ isolamento de espécies não albicans como $C$. glabrata, $C$. parapsilosis, C. krusei, C. tropicalis vem se tornando frequente ${ }^{5,6,7,8,9}$.

O tratamento da CVV depende do grau de complicação de cada caso e varia desde terapias à curto prazo e medicações orais em dose única até tratamentos prolongados por 6 meses $^{10}$.

A frequência de isolados clínicos resistentes aos agentes antifúngicos utilizados vem aumentando ao longo dos anos, principalmente em relação ao fluconazol, droga de primeira escolha no tratamento de CVV ${ }^{11,12,13}$. Parte da resistência pode ser devido à prescrição de tratamento com antifúngicos sem o devido diagnóstico de infecção por Candida ${ }^{14}$. Devido ao aumento da resistência aos antifúngicos e à toxicidade sistêmica de algumas drogas, faz-se necessário estudo de novas fontes de potenciais fármacos para o tratamento de CVV.

Em países em desenvolvimento, as pessoas de baixa renda fazem uso da medicina popular para o tratamento de infecções ${ }^{15}$. Aliado a esse fator, uma forma de prevenir a resistência a antimicrobianos seria a utilização de novos compostos que não apresentam como base os antimicrobianos sintéticos existentes ${ }^{15}$. Por esse motivo, o presente trabalho buscou investigar a atividade antifúngica de algumas plantas contra isolados de Candida spp obtidos de cavidade vaginal.

\section{Material e métodos}

\section{Obtenção dos extratos hidroalcóolicos}

A preparação dos extratos hidroalcoólicos foi realizada através do método de maceração em etanol/água, pelo período de 10 dias, utilizando álcool etílico a 50\%, sendo posteriormente filtrados e armazenados em frasco âmbar ${ }^{16}$. Alíquotas foram secas a $55^{\circ} \mathrm{C}$, até obtenção de pellets que foram ressuspendidos em $10 \mathrm{ml}$ de DMSO 1\% (Dimetilsulfóxido 1\%), e posteriormente filtradas com microfiltro $22 \mu \mathrm{m}$ (Merck KGaA, Darmstadt, Alemanha). Os extratos foram diluídos nas seguintes concentrações: folha de Cecropia spp (embaúba) - 24,3 $\mathrm{mg} \mathrm{ml}^{-}$ ; folha de Psidium spp (goiabeira) $34,4 \mathrm{mg} \mathrm{ml}$; folha de Rosmarinus spp. (alecrim) - 45,4 mg ml; folha de Byrsonima spp (muricizeiro) - 66,6 mg $\mathrm{ml}^{-}$; raiz de Byrsonima spp (muricizeiro) - 62,6 $\mathrm{mg} \mathrm{ml}^{-}$e semente de Cucurbita spp (abóbora) - 18,4 mg ml'.

\section{Micro-organismos utilizados}

Foram utilizadas duas amostras de Candida albicans, Candida 
parapsilosis, Candida tropicalis e Candida glabrata provenientes de secreção vaginal previamente identificadas e estocadas no Laboratório de Micologia da Universidade CEUMA - UNICEUMA. (Projeto aprovado pelo CEP UNICEUMA Processo no 270//10). Os inóculos foram padronizados na escala 0,5 de McFarland correspondendo a uma concentração de $10^{6}$ células $/ \mathrm{mL}^{17}$.

\section{Ensaio em meio sólido por difusão em Agar}

Os ensaios de avaliação da atividade antifúngica dos extratos foram conduzidos através do método de difusão em Agar utilizando cavidades em placas. Foram inoculados, em placas de ágar Sabouraud com cloranfenicol (Becton Dickinson $\mathrm{GmbH}$, Alemanha) $100 \mu \mathrm{l}$ da solução padronizada com auxílio de swabs estéreis. Foram feitos poços de $8 \mathrm{~mm}$ de diâmetro e adicionadas alíquotas de $50 \mu \mathrm{l}$ dos extratos brutos em cada poço. O ensaio foi realizado em duplicata e como controles foram utilizados 0 antifúngico Nistatina (128 $\left.\mathrm{mg} \mathrm{ml}^{-}\right)$e o solvente DMSO 1\%. As placas foram incubadas por 48 horas a $37^{\circ} \mathrm{C}$ e após esse período foi feita leitura dos diâmetros dos halos com auxílio de régua milimetrada.

\section{Ensaio em meio líquido}

O ensaio em meio líquido foi realizado em microplacas de 96 poços de fundo chato. $O$ extrato foi diluído em meio RPMI na proporção de 1:1 ml. Em cada microplaca foram adicionados aos poços o extrato diluído e o inóculo (1:1, v:v). Além dos poços testes foram utilizados controles de meio e inóculo (1:1, v:v); extrato diluído e salina (1:1, v:v); fluconazol e inóculo (1:1, v:v);
DMSO 1\%, meio e inóculo (0,5:0,5:1, v:v:v). As placas foram incubadas por $48 \mathrm{~h}$ a $37^{\circ} \mathrm{C}$. Os testes foram feitos em triplicata e o resultado foi obtido por quantificação da absorbância em leitor de microplacas (bioMérieux Reader 250 Version 2.0.5) no comprimento de onda de $450 \mathrm{~nm}$.

Os extratos que obtiveram resultados positivos foram submetidos à avaliação da concentração inibitória mínima (CIM)

\section{Ensaio para determinar CIM (concentração inibitória mínima)}

A concentração inibitória mínima dos extratos foi determinada pelo ensaio de microdiluição em caldo de acordo com Clinical and Laboratory Standards Institute (CLSI) padrão ${ }^{17}$. O inóculo padronizado na escala de 0.5 de McFarland foi diluído na proporção de 1:50. Os ensaios foram realizados em placas de microtitulação de poliestireno com 96 poços. Os extratos foram testados nas diluições de 1:2, $1: 4,1: 8,1: 16$ e 1:32 diluídos em meio RPMI e em seguida adicionados $100 \mu \mathrm{L}$ de suspensão celular padrão. As microplacas foram incubadas a $37^{\circ} \mathrm{C}$ por $48 \mathrm{~h}$ e após esse período foi feita leitura em leitor de ELISA (bioMérieux Reader 250 Version 2.0.5) a 450nm para determinar as CIMs. As CIMs que apresentaram inibição foram semeadas com a ajuda de um swab estéril em placas de Ágar Sabouraud dextrosecloranfenicol para determinar se 0 extrato tem caráter fungicida ou fungistático.

\section{Resultados}

Foram testados 6 extratos hidroalcóolicos obtidos de partes vegetais contra 4 espécies de Candida de isolados clínicos. O ensaio de 
difusão em ágar mostrou resultado positivo para o extrato obtido à partir da folha de Psidium spp (goiabeira), formando halos de $6 \mathrm{~mm}$ e $10 \mathrm{~mm}$ em linhagens de $C$. tropicalis. Nas demais espécies não houve inibição por nenhum dos extratos.

O ensaio em meio líquido mostrou que todos os extratos foram capazes de inibir o crescimento das linhagens de Candida, variando desde $0,8 \%$ a $96,4 \%$ de inibição. O extrato de Cecropia spp obteve a melhor atividade anti-candida, agindo contra todas as linhagens e chegando a inibir em 96,4\% 0 crescimento de $C$. tropicalis 2 (Figura $1)$.

Além do extrato obtido das folhas de Cecropia spp, os extratos da folha do Rosmarinus spp e da folha do Byrsonima spp foram os que obtiveram melhores resultados e, portanto, foi realizado o ensaio para determinar a concentração inibitória mínima destes. Os testes para avaliação da CIM mostraram que apenas o extrato da folha da Cecropia spp mostrou-se ativo, para uma das cepas de $C$. tropicalis, em concentrações menores, sendo 0,76 $\mathrm{mg} \mathrm{ml}^{-}$sua CIM, inibindo $58 \%$ do crescimento. Tal extrato foi considerado fungistático após semeadura em placa de Ágar Sabouraud, tendo em vista que apenas inibiu a proliferação das células sem causar sua morte.

O antifúngico Fluconazol, utilizado como controle positivo para os testes de microdiluição, inibiu o crescimento de $87,5 \%$ das cepas testadas. Apenas uma cepa da espécie $C$. parapsilosis se mostrou resistente ao antifúngico (Tabela 1).

\section{Discussão}

O uso de produtos de origem vegetal e animal é, historicamente, uma fonte de produtos medicinais e recentemente têm fornecido pistas para novos compostos que têm sido testados clinicamente ${ }^{18}$. Cerca de $34 \%$ dos medicamentos formulados a partir de pequenas moléculas são produtos naturais ou derivados diretos destes ${ }^{19}$.

O ensaio de difusão em ágar é o método mais utilizado para testar susceptibilidade antimicrobiana em laboratórios de microbiologia clínica, entretanto não é capaz de classificar se o efeito é fungicida ou fungistático ${ }^{20}$. Por tal motivo é necessário fazer o teste complementar de diluição, afim de determinar a $\mathrm{CIM}^{20}$. $\mathrm{O}$ presente trabalho mostrou que o único extrato a apresentar atividade anti-candida pelo método de difusão em ágar foi o de Psidium spp. Enquanto que no meio líquido todos os extratos apresentaram atividade antifúngica.

A divergência de resultados entre os dois métodos pode ser explicada pela dificuldade de difusão do extrato no meio de cultura que pode estar relacionada à sua hidrossolubilidade $\mathrm{e}$ à sua massa molecular, tendo em vista que algumas amostras apresentam característica lipofílica ${ }^{21}$.

O efeito de Psidium spp contra Candida spp vem sendo descrito ao longo dos anos, entretanto a maioria dos ensaios são realizados em linhagens de referência ${ }^{22,23,24,25}$. Alguns compostos fenólicos e flavonoides encontrados nos extratos de $P$. brownianum e $P$. guajava podem estar associados ao efeito sinérgico do extrato com o antifúngico fluconazol na inibição de Candida ${ }^{26}$.

Ao serem testados em meio líquido, todos os extratos apresentaram atividade, o que poderia ser explicado pela facilidade de se difundir em meio líquido, gerando uma maior superfície de contato. A partir deste ensaio, três extratos com a melhor atividade foram selecionados para determinação da CIM. De acordo com o critério utilizado por Menezes, Mendes e Cunha ${ }^{27}$ e 
Holetz et al. ${ }^{28}$ (Tabela 2), o extrato de Cecropia spp apresentou boa atividade antifúngica.

Plantas do gênero Cecropia, são usadas na medicina tradicional para o tratamento de infecções respiratórias, hiperlipidemias e antidiabética ${ }^{29,30,31}$. A presença de saponinas e taninos encontrados nos extratos etanólico e aquoso podem ser responsáveis pela atividade antifúngica desta planta ${ }^{32}$. Existem pouquíssimos trabalhos publicados sobre a atividade antifúngica de extratos desse gênero de planta, por este motivo são necessários novos estudos que possam contribuir para o aumento do conhecimento dessa planta e suas funções medicinais.

A planta Rosmarinus officiales já vem sendo utilizada pela medicina popular devido às suas propriedades de aliviar a dor, melhorar a digestão, estimular o fígado e a bexiga, além de combater

microrganismos patogênicos ${ }^{33}$. As atividades biológicas do extrato desta planta estão relacionadas à presença de compostos fenólicos e voláteis como carnosol e ácido rosmarínico ${ }^{34}$.

O muricizeiro (Byrsonima spp.), espécie amplamente encontrada no cerrado brasileiro, é utilizada popularmente para tratamento de diarreia, dor de garganta, feridas, inflamações, incluindo inflamação do útero, e rins ${ }^{35}$. Sua atividade antimicrobiana contra Staphylococcus aureus, S. epidermidis, Bacillus cereus, Pseudomonas aeruginosa e Escherichia coli, Klebsiella pneumoniae, Salmonella typhi, Shigella flexneri, Micrococcus luteus e Streptococcus pneumoniae já vem sendo demonstrada por alguns trabalhos ${ }^{36,37,38}$. Tal atividade contra bactérias pode estar relacionada à presença de flavonoides como catequina e epicatequina ${ }^{39}$.

\section{Conclusão}

Através do presente trabalho pode-se concluir que todos os extratos testados apresentam capacidade de inibir o crescimento de Candida em meio líquido, enquanto que no meio sólido apenas o extrato de Psidium sp, apresentou atividade anti-candida. São necessários ensaios complementares para avaliar a citotoxicidade desses extratos e entender como eles interferem nos mecanismos de virulência de Candida.

\section{Conflito de interesses}

Os autores declaram que a pesquisa foi realizada sem qualquer tipo de conflito de interesses.

\section{Referências}

1. Zeng $X$, Zhang $Y$, Zhang $T$, Xue $Y, X u H$, An R. Risk Factors of Vulvovaginal Candidiasis among Women of Reproductive Age in Xi'an: A CrossSectional Study. BioMed Research International. 2018; 2018.

2. Sobel, JD. Vulvovaginal candidosis. The Lancet. 2007; 369(9577), 1961-1971.

3. Røttingen, JA, Cameron, DW, and Garnett, GPA. Systematic review of the epidemiologic interactions between classic sexually transmitted diseases and HIV: how much really is known?. Sexually transmitted diseases. 2001; 28(10), 579597.

4. Nwadioha, SI, Egah, DZ, Alao, OO, and Iheanacho, E. Risk factors for vaginal candidiasis among women attending primary health care centers in Jos, Nigeria. Journal of clinical medicine and research. 2010; 2(7), 111-113.

5. Oliveira, SKR, Anjos, DC; Goncalves, LH, Ferro, TAF, Monteiro, SG, Figueiredo, PMS. et al. Prevalence and production of enzymes by Candida isolates from vaginal secretion samples. Rev Patol Trop. 2013; v. 42 , n. 2 , p. 161-176. 
6. Alves, MB, Silva, ÍMO, Santos, Cl, França, YR, Oliveira, SKR, M, SG, AndradeMonteiro, C. Prevalence of Candida spp. from samples of vaginal secretion and its relationship to factors associated with vulvovaginitis Rev. Investig, Bioméd., São Luís. 2015; 7:58-68.

7. Goncalves B, Ferreira C, Alves CT, Henriques M, Azeredo J, Silva S. Vulvovaginal candidiasis: epidemiology, microbiology and risk factors. Crit Rev Microbiol. 2016; 42:905-927.

8. Alizadeh M, Kolecka A, Boekhout $\mathrm{T}$, Zarrinfar H, Ghanbari Nahzak MA, Badiee P, Rezaie Matehkolaei A, Fata A, Dolatabadi S, Najafzadeh MJ. Identification of Candida species isolated from vulvovaginitis using matrix assisted laser desorption ionizationtime of flight mass spectrometry. Curr Med Mycol. 2017; 3(4): 21-25. DOI: $10.29252 / \mathrm{cmm} .3 .4 .21$

9. Nejat, ZA, Shirin F, Mehraban F, Mahtab AK, Aga FH, Azamsadat F, Masoome E e Saeideh $\mathrm{H}-\mathrm{H}$. Molecular Identification and Antifungal Susceptibility Pattern of Nonalbicans Candida Species Isolated from Vulvovaginal Candidiasis. Iranian Biomedical Journal. 2018; 22(1): 33-41

10. Dovnik, A., Golle, A., Novak, D., Arko, D., \& Takac, I. Treatment of vulvovaginal candidiasis: a review of the literature. Acta Dermatovenerol Alp Pannonica Adriat. 2015; 24(1), 5-7.

11. Bitew, A \& Abebaw, Y. Vulvovaginal candidiasis: species distribution of Candida and their antifungal susceptibility pattern. BMC women's health. 2018; 18(1), 94.

12. Mendling, W \& Brasch, J. Guideline vulvovaginal candidosis (2010) of the german society for gynecology and obstetrics, the working group for infections and infectimmunology in gynecology and obstetrics, the german society of dermatology, the board of german dermatologists and the german speaking mycological society. Mycoses. 2012; 55, 113.

13. Sobel, JD \& Sobel, R. Current treatment options for vulvovaginal candidiasis caused by azole-resistant Candida species. Expert Opinion on Pharmacotherapy. 2018; 1-7.

14. dos Santos Brandão, LD, Boniek, D, de Resende Stoianoff, MA, da Mata, FMR, de
Azevedo, PRM, Fernandes, JV, \& Andrade, VS. Prevalence and antifungal susceptibility of Candida species among pregnant women attending a school maternity at Natal, Brazil. Letters in applied microbiology. 2018; 67(3):285-291.

15. Rojas, JJ, Ochoa, VJ, Ocampo, SA, \& Muñoz, JF. Screening for antimicrobial activity of ten medicinal plants used in Colombian folkloric medicine: A possible alternative in the treatment of nonnosocomial infections. BMC complementary and alternative medicine. 2006; 6(1), 2.

16. MARTINS, TVF et al. Atividade antibacteriana de Brassica oleracea var. capitata em modelos experimentais in vitro. Monografias ambientais. 2012; 9 (9), 2088- 2100 .

17. Wayne, PA. USA: CLSI; 2012. Clinical and Laboratory Standards Institute. Performance Standards for Antimicrobial Susceptibility Testing. Twenty-Second Informational Supplement. CLSI Document M100-S22.

18. Harvey, AL, Edrada-Ebel, R, \& Quinn, RJ. The re-emergence of natural products for drug discovery in the genomics era. Nature Reviews Drug Discovery. 2015; 14(2), 111.

19. Newman, DJ, \& Cragg, GM. Natural products as sources of new drugs over the 30 years from 1981 to 2010 . Journal of natural products. 2012; 75(3), 311-335.

20. Balouiri, M, Sadiki, M, \& Ibnsouda, SK. Methods for in vitro evaluating antimicrobial activity: A review. Journal of pharmaceutical analysis. 2016; 6(2), 7179.

21. Alves, E.G. et al. Estudo comparativo de técnicas de screening para avaliação da atividade antibacteriana de extratos brutos de espécies vegetais e de substâncias puras. Química Nova. 2008; 31 (5), 12241229.

22. Fernandes, MRV, Dias, ALT, Carvalho, RR, Souza, CRF, \& Oliveira, WP. Antioxidant and antimicrobial activities of Psidium guajava L. spray dried extracts. Industrial Crops and Products. 2014; 60, 39-44.

23. Costa, GM, Endo, EH, Cortez, DAG, UedaNakamura, T, Nakamura, CV, \& Dias Filho, BP. Effect of plant extracts on planktonic growth and biofilm of Staphylococcus 
aureus and Candida albicans. Int. J. Curr. Microbiol. App. Sci. 2015; 4(6), 908-917.

24. Abbas, B., \& Fatima, T. Evaluation of antibacterial and antifungal activities of Cymbopogon citratus \& Psidium guajava from sialkot origin. Evaluation. 2018; 1, 155-163.

25. Sangalli, J, Júnior, EGJ, Bueno, CRE, Jacinto, RC, Sivieri-Araújo, G, Gomes Filho, JE, ... \& Junior, E. D. Antimicrobial activity of Psidium cattleianum associated with calcium hydroxide against Enterococcus faecalis and Candida albicans: an in vitro study. Clinical oral investigations. 2018; 22(6), 2273-2279.

26. Morais-Braga, MFB, Sales, DL, Carneiro, JNP, Machado, AJT, dos Santos, ATL, de Freitas, MA, ... \& Souza, DS. Psidium guajava L. and Psidium brownianum Mart ex DC.: Chemical composition and antiCandida effect in association with fluconazole. Microbial pathogenesis. 2016; 95, 200-207.

27. Menezes, EA; Mendes, L.G.; Cunha, FA. Antifungal resistance of Candida tropicalis isolated in the State of Ceará. Revista da Sociedade Brasileira de Medicina Tropical. 2009; 42 (3), 354-355.

28. Holetz, F.B. et al. Screening of Some Plants Used in the Brazilian Folk Medicine for the Treatment of Infectious Diseases. Mem. Inst. Oswaldo Cruz, Rio de Janeiro, v. 97, n. 7, p. 1027-1031, oct. 2002.

29. Silva, MABD, Melo, LVL, Ribeiro, RV, Souza, JPMD, Lima, JCS, Martins, DTDO, \& Silva, RMD. Ethnobotanical survey of plants used as anti-hyperlipidemic and anorexigenic by the population of Nova Xavantina-MT, Brazil. Revista Brasileira de Farmacognosia. 2010; 20(4), 549-562.

30. de Souza, CD, \& Felfili, JM. Uso de plantas medicinais na região de Alto Paraíso de Goiás, GO, Brasil. Acta Botanica Brasilica. 2006; 20(1), 135-142.

31. Rodrigues, VEG., \& Carvalho, DD. Levantamento etnobotânico de plantas medicinais no domínio do cerrado na região do Alto Rio Grande-Minas Gerais.
Ciência e Agrotecnologia. 2001; 25(1), 102-123.

32. Costa, JCFD, \& Hoscheid, J. Perfil fitoquímico e avaliação da atividade antimicrobiana de extratos aquoso e etanólico de folhas de Cecropia pachystachya. Revista Fitos. 2018; 12 (2), 175-185.

33. Erdogrul, ÖT. Antibacterial activities of some plant extracts used in folk medicine. Pharmaceutical Biology, 2002; 40(4), 269273.

34. Ribeiro-Santos, R, Carvalho-Costa, D, Cavaleiro, C, Costa, HS, Albuquerque, TG, Castilho, MC, ... \& Sanches-Silva, A. A novel insight on an ancient aromatic plant: The rosemary (Rosmarinus officinalis L.). Trends in Food Science \& Technology. 2015; 45(2), 355-368.

35. Oliveira, RLCD, Scudeller, VV, \& Barbosa, RI. Use and traditional knowledge of Byrsonima crassifolia and B. coccolobifolia (Malpighiaceae) in a Makuxi community of the Roraima savanna, northern Brazil. Acta Amazonica. 2017; 47(2), 133-140.

36. Alves, TMDA, Silva, AF, Brandão, M, Grandi, TSM, Smânia, EDFA., Smânia Júnior, A, \& Zani, CL. Biological screening of Brazilian medicinal plants. Memórias do Instituto Oswaldo Cruz. 2000; 95(3), 367373.

37. Pereira, VV. Estudo fitoquímico de Byrsonima coccolobifolia Kunth (Malpighiaceae) e de atividade biológica de espécies do gênero Byrsonima [dissertação] Minas Gerais: Universidade Federal dos Vales do Jequitinhonha e Mucuri; 201.

38. Martinez-Vazquez, M, GonzalezEsquinca, AR, Cazares, LL, Moreno, MG, \& García-Argaez, AN. Antimicrobial activity of Byrsonima crassifolia (L.) HBK. Journal of ethnopharmacology. 1999; 66(1), 79-82.

39. Kuster, VC, \& Vale, FH. Leaf histochemistry analysis of four medicinal species from Cerrado. Revista Brasileira de Farmacognosia. 2016; 26(6), 673-678. 